\title{
Deep Learning for Medical Materials: Review and Perspective
}

\author{
Yangjie $\mathrm{Qi}^{1}$ and Chang $\mathrm{Liu}^{2}$ **
}

\begin{abstract}
Deep learning and similar computational research approaches have cooperated with materials research for years. Especially in the last few years, with the fast evolution of machine learning and deep learning algorithms, a novel branch for material research is presented to be recognized, learned, practiced, adapted and perfected. Different conventional computational modeling methods, the deep learning approach assists material science and engineering from the aspect of data processing and analysis, rather than simulate the reality. However, mistakes always exist in all less explored concepts and developments. Here, in order to offer a better instruction of deep learning for materials scientists, several recent works on deep learning based material research were reviewed. The application of deep learning in material research is introduced and discussed. As an example of both general material and advanced material, medical material was discussed for the impact and future of deep learning methods. At last, we provided several insights on future work for both deep learning scientists, data scientists and materials scientists.
\end{abstract}

Keywords: Deep Learning; Materials Research; Medical Material. Received date: 21 August 2020; Accepted date: 14 Februray2021.

Article type: Review article.

\section{Introduction}

Medical material has grown greatly together with modern chemistry, material science and medical science. From the basic metallic alloy to medical glass, from the plastic cloth on the surgery table to advanced tissue regeneration hydrogel, the field of medical material covers almost all subjects in material science and engineering. Since the material relates to human health, most countries have their regulators or regulations such as FDA (U.S. Food and Drug Administration), EU (CE marking Directorate-General for Internal Market, Industry, Entrepreneurship and SMEs, European Union), CMDCAS (Canadian Medical Devices Conformity Assessment System), NMPA (National Medical Products Administration, China), MFDS (Ministry of Food and Drug Safety, Korea), TGA (Therapeutic Goods Administration, Australia), standards such as ISO, GB, JIS. However, these regulations are mostly focusing on general materials such as medical alloys (such as nitinol, stainless steel, titanium) and general plastics (such as ABS, PDMS, PU). The steps of pre-clinical research, clinical trial and post-market safety monitoring period for novel advanced material are too long for the invention cycle of new

\footnotetext{
${ }^{1}$ Department of Electrical and Computer Engineering, University of

Dayton, Dayton, OH 45469, USA.

${ }^{2}$ Adva-Nano LLC, Centerville, $\mathrm{OH} 45458$, USA.

*E-mail: changliu0902@gmail.com (C. Liu)
}

material and the career pace of young investors. It usually takes more than 10 years on average for a drug to be marketed from the laboratory. ${ }^{[1,2]}$ More years are necessary to monitor implants such as artificial organs, tissue scaffold, stent and artificial tissue to verify the material's safety in a long-term period. As a result, since the validation process is unavoidable, faster approaches in material research and development could help to accelerate the commercialization cycle.

The computational method is one of the main approaches to reduce the expense of the costly biomedical experiments. Conventional computational methods in material science are mainly atomistic simulation, molecular modeling, and ab initio (or de novo) type simulation. ${ }^{[3-5]}$ With the application of high-performance computers or supercomputers, the fascinating computing power enables researchers to perform accurate or large quantity atomistic simulations. Meanwhile, the generated data is increasing dramatically compared to the traditional laboratory experimental approach. Currently, processing and analyzing such amounts of data are well managed. However, there is a huge amounts of data at the pharmacotherapeutics field. Bio-informatics is such a subbranch explored from interpreting a large amount of data. The requirement of dealing with billions of genetic sequences in DNA (deoxyribonucleic acid), RNA (ribonucleic acid), structure of proteins, and various bioactive chemicals have pushed biomedical researchers to various mathematical tools. These mathematical tools later evolved together with data 
science, artificial intelligence, network analysis, and in silico simulation methods. Back to material science, a similar evolution path is expected.

During the last several years, deep learning (DL) and other machine learning methods, such as neuron inspired spiking neural networks (SNN), ${ }^{[6]}$ have been applied to various material science topics in various ways. ${ }^{[7]}$ In this review, we presented an illustrative introduction of DL for material scientists in section 2 . Then, in section 3 , the research life cycle of general materials and advanced materials were classified into six subjects for ease of discussion. An in-depth discussion on each subject was provided as well. In section 4 , insights for current studies were discussed. Section 5 used medical material as an example shows the cooperation of DL and materials research. At last, we summarized the current problems and offered a perspective for researchers.

\section{Background}

DL comes from the study on Artificial Intelligence (AI), which intends to allow computer systems to perform humanlevel intelligence. So far, no system that has achieved general intelligence. But we can make system performance the same category of tasks extremely well, even better than human, in fields such as image recognition ${ }^{[8]}$ and game strategy. ${ }^{[9]}$ Most of those systems are powered by machine learning algorithms. Among various machine learning algorithms, neural networks $(\mathrm{NN})$, sometimes referred to as artificial neural networks (ANN), are one of the significant groups. The neural network was proposed in 1943 and has experienced several waves of evolution. And DL is the most successful group in current evolution (Fig. 1).

In 2015, LeCun et al. ${ }^{[10]}$ defined DL methods as representation learning methods with multiple levels of representation, obtained by composing simple but non-linear modules that each transform the representation at one level into a representation at a higher, slightly more abstract level. As time goes on, the DL field is kept developing and evolving fast. However, it can be categorized as supervised, semisupervised, and unsupervised as shown in Fig. 1b. This

a

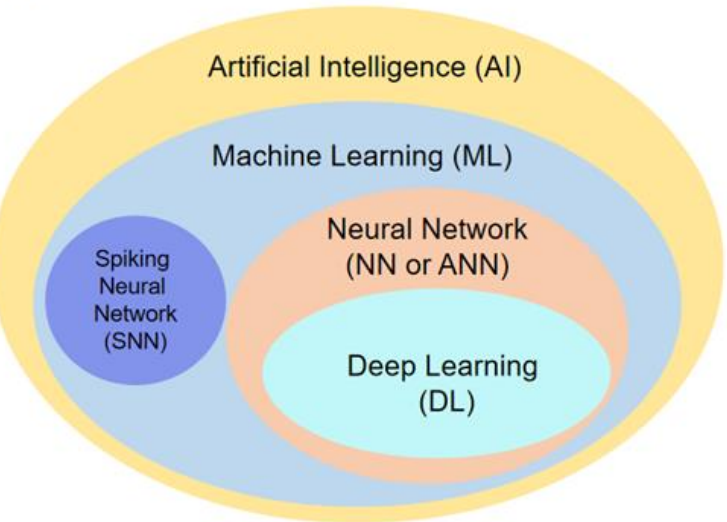

categorization method is also used in machine learning. The key difference among those three categories is the training data type used for learning.

In supervised learning, all the data used for training are labeled clearly. The system gets a set of inputs and corresponding labels to train the inside network parameters for better prediction of the outputs. After training, the system can correctly predict the label of new data. Convolutional Neural Networks (CNNs), and Recurrent Neural Networks (RNNs) are two widely used supervised learning methods. CNNs are widely used in image and video processing applications. The key configuration of CNNs is a series of convolutional layers, which can efficiently abstract the features of data, especially data that have locality of reference. For example, using twodimensional (2D) cross-section images and corresponding results to predict the effective thermal conductivity of threedimensional (3D) composites. ${ }^{[11]}$ RNNs are generally used in natural language processing and speech recognition applications. RNNs show outstanding performance in using sequential data or time-series data to solve ordinal or temporal problems, such as sensing behavior prediction of the wearable device. ${ }^{[12]}$

For unsupervised learning, the system gets unlabeled data and discovers the underlying features and hidden connections/interactions of those data. This approach is generally used for clustering, dimension reduction and data enhancement. The commonly used unsupervised learning approach includes Auto Encoders (AEs), Restricted Boltzmann Machines (RBMs), and Generative Adversarial Networks (GANs). For example, GANs are generally used for data enhancement purposes. GANs consist of a discriminator and a generator. The generator is trained to generate new data, while the discriminator is trained to judge if the given data is the real data or is made by the generator. Microstructure generation is one of the most applied fields for unsupervised learning methods. $\mathrm{Li}$ et al produced high-quality microstructures from GAN for further prediction of physical properties. ${ }^{[13,14]}$

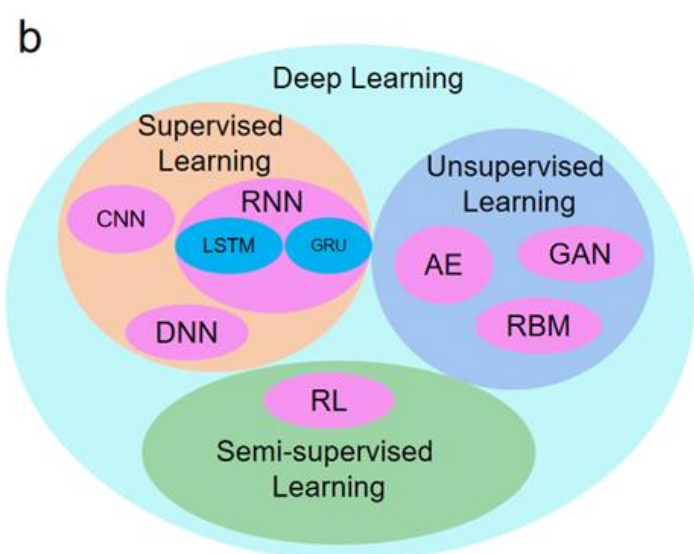

Fig. 1 (a) Deep learning in the context of AI and (b) the content of deep learning. 
Semi-supervised learning also referred to as reinforcement learning (RL), is trained by semi-label data. The result of an RL system is generally a score instead of a clear label. RL is a state-based system, the results depend on previous actions. The most famous RL system is the AlphaGo of Google DeepMind. ${ }^{[9]}$ The RL systems generally consist of an agent and environments. The agent makes a sequence of decisions, while environments may change statues and/or give rewards to the agent. The target of training an RL system is to make the agent achieve the highest rewards. In other words, let the agent make the right decision in every statue of the environment.

In previous studies, the use of DL in material science is mainly based on supervised learning, mostly CNN. Unsupervised learning methods, such as GAN and RBM, are also used. Based on our knowledge, the RL system has not been widely applied in our community. But we believe it is a very powerful tool and potentially to be applied in the future. In an RL system, the environment is initialized into a specific state. The agent would make a sequence of decisions, which may change the state of the environment, and based on the decision and status change, the environment may give the agent rewards. These processes are very similar to what we do in materials research. Such a feedback loop for justification is potentially an integrated toolbox. In the rest of the paper, we will mainly focus on the supervised DL which is widely used in the material science and engineering field.

\subsection{Deep learning algorithms}

In a supervised DL system, we first need to collect a large amount of data with labels to create a dataset. Then the data will be input into the system and expect it can generate results that can match the corresponding labels, but this is unlikely to happen until the system is trained properly. Next, an object function is configured to compute errors, which measure the difference between the outputs and labels. At last, the DL algorithm will adjust the internal parameters (weights) of the system to minimize the errors. The above procedure may repeat tens or even hundreds of times until the errors are converged.

The algorithm used in the system to adjust weights is called gradient descent, which computes a gradient vector that, for each weight, indicates by what amount the error would increase or decrease if the weight were increased by a tiny amount. The weight vector is then adjusted in the opposite direction to the gradient vector. ${ }^{[10]}$ There are three variations of the gradient descent, they are batch gradient descent, stochastic gradient descent (SGD), and mini-batch gradient descent. ${ }^{[10]}$ Now the most widely used is the mini-batch gradient descent, as it finds a balance between the robustness of SGD and the efficiency of batch gradient descent. ${ }^{[15]}$

Some optimization techniques are designed to further improve the overall performance of the gradient descent. Momentum is a technique for accelerating gradient descent in the relevant direction and dampens oscillations. ${ }^{[16]}$ AdaGrad
(Adaptive Gradient Algorithm) ${ }^{[17]}$ is an algorithm that adapts the learning rate based on the parameters. Ada Delta ${ }^{[18]}$ and RMSprop $^{[19]}$ are two extensions of AdaGrad. Adaptive Moment Estimation (Adam) ${ }^{[20]}$ combines the idea of momentum and RMSprop. One of the above can be applied to the training algorithms, however, there is no best optimization method. Each of the above methods outperforms others in different applications. If a new dataset or application is being studied, it is better to try several training algorithms and techniques. And Adam is the one to start with, as it shows the best overall performance in several benchmarks. ${ }^{[21]}$

Another two important training techniques of DL are dropout ${ }^{[22]}$ and batch normalization. ${ }^{[23]}$ Dropout is a commonly used regularization technique to prevent the deep network from overfitting and provides a way of approximately combing exponentially many different neural network architectures efficiently. ${ }^{[17]}$ It is simply deployed by random removal of part of the connections of the network during the training process while showing incredible performance. Batch Normalization normalizes the inputs of each layer, so the network will have a mean output activation of zero and a standard deviation of one. By applying this technique, it makes the network train faster in higher learning rates. It also makes the network less sensitive to the initialize parameters and provides some regularization. The dropout and batch normalization are proven to be useful in the DL algorithm and the majority of applications. Both of the two techniques are highly recommended to start with.

\subsection{Deep learning networks}

There is a lack of count about how many different network architectures in DL. People always set up their version of networks for different applications and their own needs. Three frequently used networks are discussed below, CNN, RNN, and GAN. The relation and selection of supervised learning methods are illustrated in Fig. 2 as well.

\subsubsection{CNN}

Since 2012, the winners for the ImageNet challenge are all $\mathrm{CNN}$. All the CNN networks are the combination of a different number of convolution layers, pooling layers, and fully connected (FC) layers. Some famous networks, such as AlexNet ${ }^{[24]}$, GoogLeNet ${ }^{[25]}$, ResNet ${ }^{[26]}$ are all the winners for the ImageNet challenge. ${ }^{[2]]}$ The accuracy increase as the network goes deeper and computational cost goes up. But it does not mean the deeper the better. A deeper network also needs more careful configuration. It is always a good idea to adjust a well-designed network and apply it to new applications. ${ }^{[28]}$

\subsubsection{RNN}

The RNN is widely used for sequence data or time-series data. There is no obvious change with the Deep RNN with the traditional recurrent neural network except the network goes deeper. Long short-term memory (LSTM) ${ }^{[29]}$ or Gated 


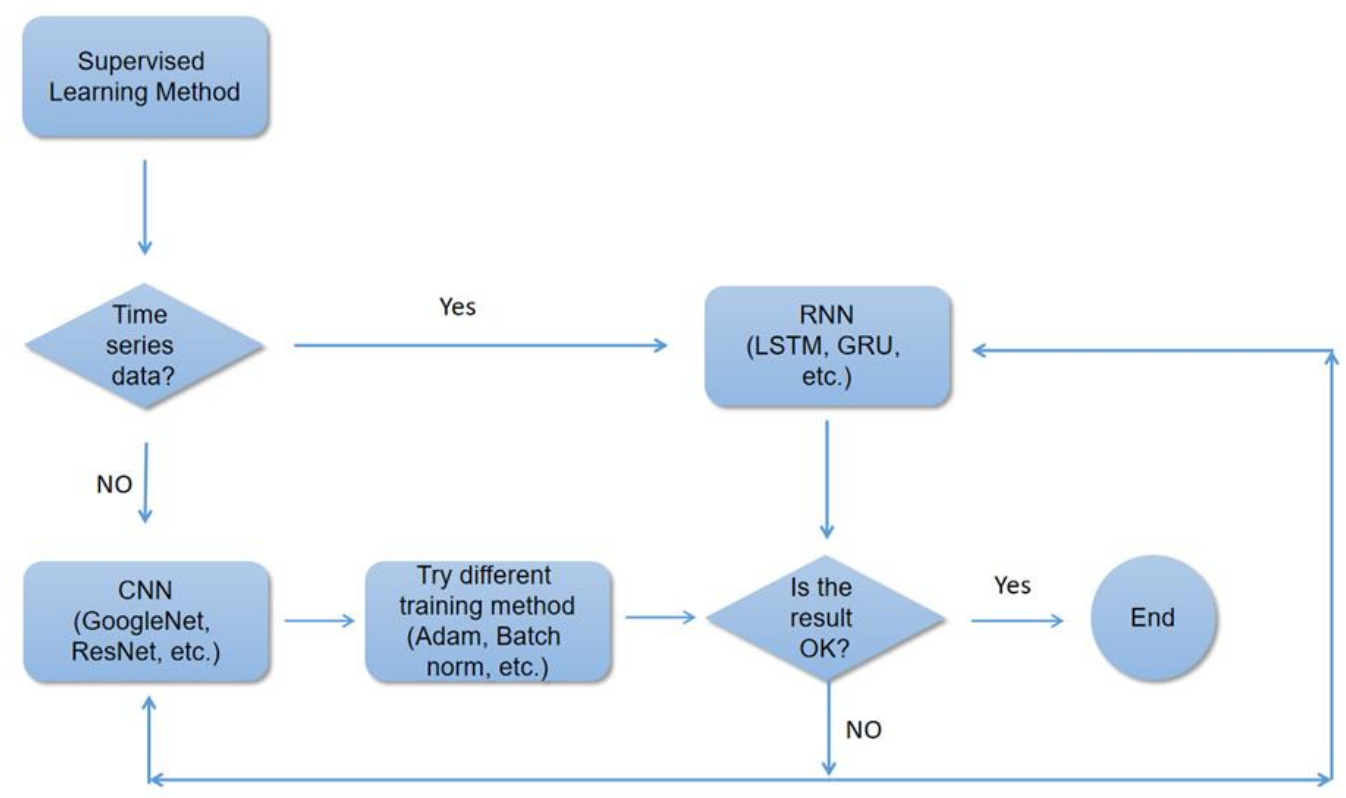

Fig. 2 Flowchart for selection and process of supervised learning method.

recurrent unit (GRU) ${ }^{[30]}$ are commonly used RNN architectures. The GRU requires fewer parameters, which makes training processing of the network faster, while LSTM provides better performance for some applications. ${ }^{[31]}$

\subsubsection{GAN}

With DL getting incredible success in many domains, the limitation of DL is also obvious, that is it relies on a large amount of labeled training data. However, the labeled data is hard or expensive to access in many domains like medicine, robotics. Generative Adversarial Networks (GANs) ${ }^{[32]}$ are developed to automatically generate larger and richer datasets from a little initial training dataset. The networks are quickly extended and deployed in many applications. ${ }^{[28,33-36]}$ GAN configures two adversaries: a discriminator and a generator, both are typically modeled as deep neural networks. Both discriminator and generator are trained in parallel. The generator tries to create new data similar to the original data, while the discriminator attempts to judge the data is produced by the generator or from the original datasets.

\subsection{Dataset}

While the algorithms are considered as the engine of DL, the dataset is the fuel of this engine. Dictionaries and encyclopedias are the commonest datasets in our life. Today, besides information technology, many other fields have established their database and dataset. For example, the genome datasets, asteroid datasets, and there are datasets for chemistry, physics and material science and engineering. Over the decades, researchers established various large or small datasets based on theoretical or experimental methods. With the recent recognition of the significance of big data and artificial intelligence methods, small datasets are being expanded into larger ones with a more organized structure. We summarized some commonly used datasets, specialized datasets, non-profit datasets organizations and commercial organizations in table 1.

The aforementioned datasets usually contain enough data for a not-bad DL training work. Besides, a small dataset could provide useful data as well, such as MD-17[53], NMD-18 ${ }^{[54]}$, BA10-18 ${ }^{[55]}$ W-14 ${ }^{[56]}$, BFCC-13 ${ }^{[57]}$, H2O-13 ${ }^{[58]}$, ETIM17@Dryad ${ }^{[59]}$. They are ab initio, density-functional theory (DFT) based small dataset for special chemicals or alloys such as H2O, Al-Ga-In, AlMg, AgPt, and tungsten. Some of the datasets are focusing on special properties of the material such as electronic transportation, electronic structure (band gap) and molecular dynamics trajectories.

Despite these datasets being used in published research works and projects, many datasets are organized by governments or related organizations such as the database of NASA (The National Aeronautics and Space Administration) ${ }^{[60]}$, NIST (The National Institute of Standards and Technology) ${ }^{[61]}$, DOE (Department of Energy) ${ }^{[62]}$, OSTI (The Office of Scientific and Technical Information) ${ }^{[63]}$, Materials Data Facility ${ }^{[64]}$ and the Materials Project ${ }^{[65]}$ of the United States, NIMS databases ${ }^{[66]}$ of Japan, National Science Technology Infrastructure ${ }^{[67]}$ of China and the aforementioned FAIR-infrastructure ${ }^{[37]}$ of European Union. Commercial dataset search engines and datasets such as Google dataset search, re3data.org, open data portal watch, Total Materia, Matweb and ASM international offer either the data or a searching platform.

\subsection{Medical material research cycle}

Typically, medical materials are classified into two categories: general medical device material and advanced material. General medical device materials consist of most of the usage volume with a wide span from common polypropylene and 
Table 1. A summary of datasets for materials science and engineering.

\begin{tabular}{|c|c|c|c|c|c|c|}
\hline Name. & Full Name & Organization & Subject & Dataset Size & Description & Ref. \\
\hline $\begin{array}{c}\text { MINC of } \\
\text { Opensurface }\end{array}$ & $\begin{array}{l}\text { Materials in Context } \\
\text { Database }\end{array}$ & Cornell University & Images & $\begin{array}{c}104,505 \text { for } \\
\text { materials }\end{array}$ & Image and surface analysis. & [39] \\
\hline NoMaD & $\begin{array}{l}\text { Novel Materials } \\
\text { Discovery }\end{array}$ & $\begin{array}{l}\text { FAIR-Data } \\
\text { Infrastructure }\end{array}$ & Materials & $\begin{array}{l}10,874,731 \\
\text { entries }\end{array}$ & Various. & [41] \\
\hline $\mathrm{EPiC}$ & $\begin{array}{c}\text { Environmental } \\
\text { Performance in } \\
\text { Construction Database }\end{array}$ & $\begin{array}{l}\text { University of } \\
\text { Melbourne }\end{array}$ & Materials & & $\begin{array}{l}\text { Concrete and construction } \\
\text { material properties. }\end{array}$ & [43] \\
\hline NanoMine & & Duke University & Materials & & $\begin{array}{l}\text { Finite element analysis (FEA) } \\
\text { based online platform of } \\
\text { materials genome prediction } \\
\text { for polymer nanocomposites. }\end{array}$ & {$[44]$} \\
\hline PPPDB & $\begin{array}{c}\text { Polymer Property } \\
\text { Predictor and Database }\end{array}$ & $\begin{array}{l}\text { University of } \\
\text { Chicago, } \\
\text { Northwestern } \\
\text { University, } \\
\text { Argonne NL }\end{array}$ & Polymer & & $\begin{array}{c}\text { Prediction of structural } \\
\text { thermodynamic and dynamic } \\
\text { properties of functional } \\
\text { polymers }\end{array}$ & {$[45]$} \\
\hline \multicolumn{2}{|c|}{ Khazana Polymer/VASP } & $\begin{array}{l}\text { Georgia Institute } \\
\text { of Technology }\end{array}$ & $\begin{array}{l}\text { Chemistry, } \\
\text { Materials }\end{array}$ & & $\begin{array}{l}\text { A platform to store structure } \\
\text { and property data created by } \\
\text { atomistic simulations and } \\
\text { tools to design materials by } \\
\text { learning from the data. }\end{array}$ & [47] \\
\hline ATCT & $\begin{array}{c}\text { Active } \\
\text { Thermochemical } \\
\text { Tables }\end{array}$ & $\begin{array}{l}\text { Argonne National } \\
\text { Laboratory }\end{array}$ & Materials & $\sim 1600$ & $\begin{array}{c}\text { Experimental and theoretical } \\
\text { data based thermochemical } \\
\text { values. }\end{array}$ & [48] \\
\hline AtomWork & $\begin{array}{l}\text { Inorganic Material } \\
\text { Database }\end{array}$ & $\begin{array}{l}\text { National Institute } \\
\text { for Materials } \\
\text { Science }\end{array}$ & $\begin{array}{l}\text { Chemistry, } \\
\text { Materials }\end{array}$ & $\begin{array}{r}82,000 \text { cry } \\
\text { properti }\end{array}$ & $\begin{array}{l}\text { al structure, } 55,000 \text { material } \\
15,000 \text { phase diagrams. }\end{array}$ & [49] \\
\hline \multicolumn{7}{|c|}{ Other specialized databases } \\
\hline $\begin{array}{l}\text { NIST- } \\
\text { JANAF }\end{array}$ & $\begin{array}{l}\text { Thermochemical } \\
\text { tables }\end{array}$ & NIST & $\begin{array}{l}\text { Inorganic } \\
\text { materials }\end{array}$ & 1,796 & & {$[50]$} \\
\hline \multicolumn{2}{|c|}{ Index of material properties } & NIST & & & $\begin{array}{l}\text { Cryogenic to room } \\
\text { temperature properties of } \\
\text { solid materials }\end{array}$ & {$[51]$} \\
\hline \multicolumn{2}{|c|}{$\begin{array}{c}\text { Property data summaries for advanced } \\
\text { materials }\end{array}$} & NIST & & \multicolumn{2}{|c|}{$\begin{array}{l}\text { Thermal, mechanical, structural and chemical } \\
\text { properties of materials from published data }\end{array}$} & {$[52]$} \\
\hline
\end{tabular}


ABS to specialized polyurethane and PDMS. The medical industry normally deals with these general medical materials with a designed manufacturing chain all over the world. The production of these materials is in a large volume, on the other hand, the improvement of these materials is slow. On the contrary, the advanced material evolves fast but is mostly limited in laboratories. As a result, the research life cycle for general medical material is simpler than that of advanced medical material.

The requirement for general medical device material is normally simply, non-harmful while in use or less harmful compared to its benefit. As a result, even with minor toxicity, cyanoacrylate adhesives are widely used as tissue glue. ${ }^{[68,69]}$ No need to mention general ABS, polyurethane, PDMS and epoxy based medical devices, the requirements for general medical material are easy to be satisfied. Besides, the materials (composites) from an approved medical device could be directly used for new devices. This rule not only accelerates the development of medical devices, but it also pushes minority materials to be produced in large quantities. The life cycle difference for general medical material research is shown below (Fig. 3).

Researchers and engineers are trending to change the design rather than seek any new material in R\&D. For example, endoscopic surgery asks for sophisticated and exquisite design for the non-invasive or micro-invasive operation to minimize the painfulness and manage the risk of infection. Currently, engineers are struggling with conservative materials for complex designs rather than try out new extreme material to simplify the design. With more complex mechanics, the chance to fail is higher. On the other hand, exploring and trying out new materials is costly by money and time. So, the need to balance the research life cycle cost and figure out the appropriate material is presented.

The advanced medical material is represented by biomaterials such as hydrogels, hydroxyapatites, biodegradable polyesters (some aliphatic polyesters) and biocompatible polymers and ceramics, post-treated animal organ tissue and

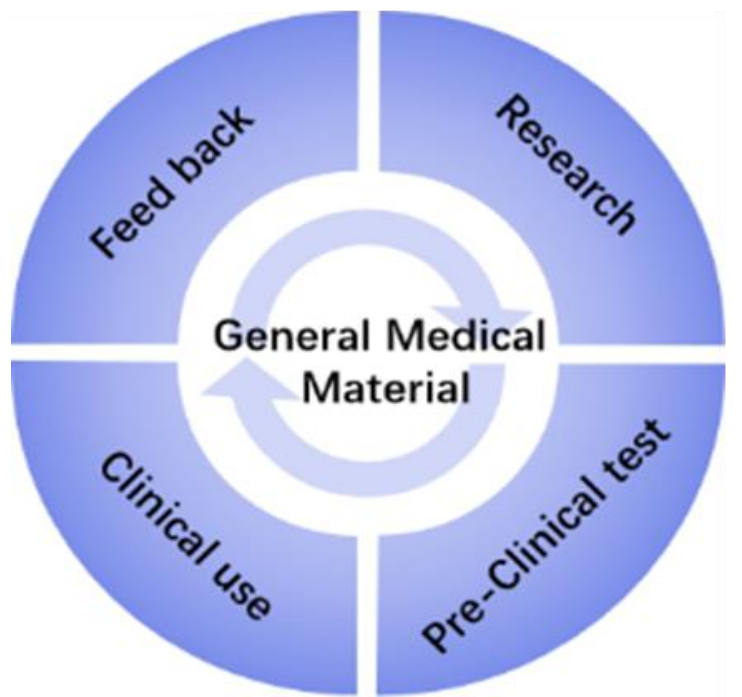

the emerging brain-machine interface material. These materials mostly only exist in scientific laboratories due to their novelty, cost and lack of in vivo test or long-term effect investigation. For instance, the emerging triboelectric nanogenerator (TENG) and piezoelectric nanogenerator (PENG) materials have drawn great attention for inventing self-powered implantable devices. ${ }^{[70]}$ However, these materials usually consist of multiple components and are structurally sophisticated. The research process on these advanced medical materials is familiar to scientists as shown in Fig 3. Different from the general medical material, advanced medical material changes and evolves more frequently due to its experimental nature. The fast-evolving pace of these advanced materials brings an additional problem: lacking comprehensive characterization of the material and which makes it hard to be organized into any database. The emerging computational methods, programs, kits, and platforms might be one solution. A standardized simulation program could provide a series, organized and comprehensive data of materials in any given condition. As a result, the data generated from simulators could cover all aspects of the material, theoretically. However, it brings another problem, how to deal with such a large amount of data. This is why we need to introduce the method for a large amount of data, deep learning.

\section{Deep learning in the research cycle}

DL scientists think differently from material scientists when facing the same question. In order to have a better understanding of how DL could be used in materials science and engineering, we organized this section by research cycle (research purpose), rather than research object (application).

\subsection{Problem identification}

Problem identification is one of the most common purposes for material scientists using DL methods. With preacquired data, DL might be a good way to figure out the abnormalities among the unorganized raw data. Medical imaging science is a good example to illustrate this. With the

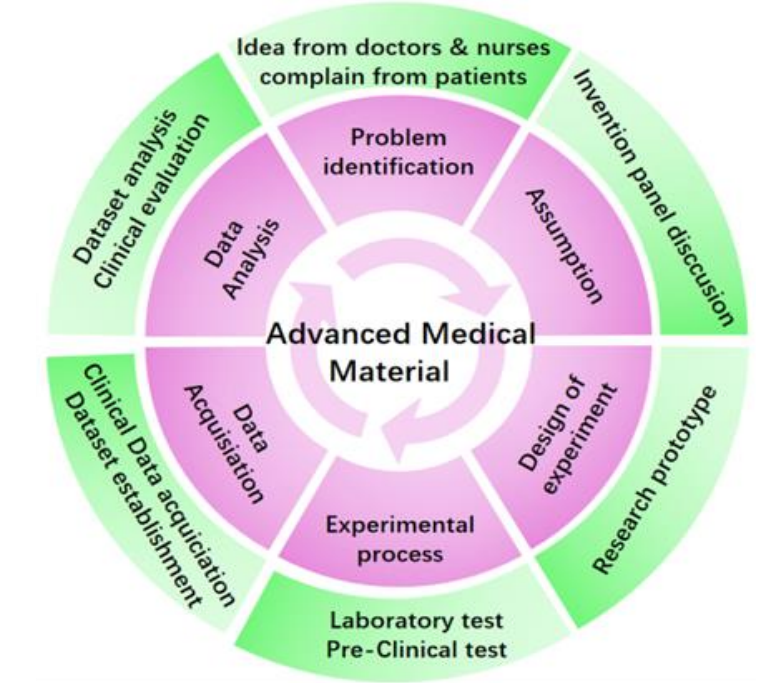

Fig. 3 The research life cycle for general medical materials and advanced medical materials. 
patient's imaging data, a well-trained DL program could find the abnormal site in the image within a glance. Similar to material science and engineering, a 3D printing structure may contain abnormal micro-cracks or unexpected voids that are hard to be identified or will cost a large amount of human effort to figure out. In this case, a well-trained DL program may be a more cost-effective way to examine $3 \mathrm{D}$ printed objects. ${ }^{[71]}$ At the same time, the industry may prefer to identify unlabeled defects in their product. Since problem identification is a part of the research life cycle, it functions similarly to data analysis but with a different purpose. From the aspect of deep learning, we may consider data analysis (result analysis) as labeled data analysis which is subject to supervised learning methods. However, the problem identification process may be considered for unsupervised learning methods. ${ }^{[72]}$

\subsection{Assumption}

The assumption is a very abstract activity that originates from logical thinking and conceptualizing. DL is different from conventional modeling processes which specify each step required to solve the problem. ${ }^{[73]} \mathrm{DL}$ is an approach of seeking a method to help computers build their algorithm based on the training data offered, despite the nature of the material's information. Since general artificial intelligence is still far away from general artificial intelligence, we may not be able to depend on programs to 'think' out a new equation, a new theorem, a new reaction, or a new hypothesis.

\subsection{Design of experiment}

It seems the design of the experiment will cost a similar brain power as the assumption, however, the powerful Cluster Analysis of DL could help a lot in the designing work. The cluster analysis helps in organizing the parameters and which reduces the dimension of the experiment. For example, a welltrained image analysis network and prediction model could dynamically adjust $3 \mathrm{D}$ printing parameters and make the different layers to be self-adapted. ${ }^{[7,75]}$

\subsection{Experimental process}

Once we use DL in our research cycle, monitoring the effectiveness of the chosen model is necessary for obtaining a reliable result. Usually, the initial DL model may not be perfect for the designed question. We should keep monitoring the model closely with new data collected and updating the model if necessary. There are also many attempts to simplify well established commercial software by training the network with simulated results. For example, FEA and DFT based software are being used to generate datasets. By analyzing networks of inputs and outputs, a prediction model could be established for a faster result. However, since the DL network is sensitive to the reliability of the dataset, it's questionable to use simulated results rather than experimental ones. ${ }^{[76,77]}$

\subsection{Data acquisition}

The data acquisition process consists of choices of data type and method of acquisition. The aim of data acquisition is to establish a processable dataset for DL type or other analysis. As a result, the data should be processable or accessible by the program.

The method of data acquisition problem is mainly for the time series based continuous data recording process. ${ }^{[78]}$ In many laboratory tests, researchers have to choose an appropriate interval to record the data, otherwise, the program could crash easily or the obtained data could be meaningless. However, when it comes to DL, parameters such as interval, sequence length, and digits become not that important. DL researchers look at the dataset in a different way. Establishing appropriate data formats is the fundamental of a successful system. There is no doubt that the more data you get, the better performance you can expect. In a conclusion, we should collect as much data as possible.

DL could be used to generate data. ${ }^{[79]}$ Inverse networks based on autoencoders show a good ability to achieve this. ${ }^{[80]}$ GAN is more widely used for generating datasets in DL fields and potentially can be deployed in our community.

\subsection{Data analysis}

Data analysis is the main use of DL in material science and engineering. ${ }^{[8]}$ However, the responsibility for material scientists is much less than in other areas such as database establishment. The material scientist can do few efforts in cleaning the data and constructing the network. It's our responsibility to understand which kind of data analysis is needed and well communicate with deep learning scientists on how to perform the network training.

Although the same for data analysis, the approach of using DL varies. Liu et al. firstly trained the DL network forwardly and then used the related parameters to optimize fabrication parameters with DL backwardly. ${ }^{[76]}$ Dynamic analysis using trained DL models could be applied for in situ monitoring purposes. ${ }^{\left[{ }^{[2]}\right]}$ Slicing material and analyzing the microstructure image with DL could help in reconstructing 3D structure and predict its properties ${ }^{[83]}$ Classification of results could simply be used for detecting and predicting abnormal events. ${ }^{[84]}$

\subsection{From the aspect of DL}

DL scientists value three steps in practicing: pre-processing, model building, model evaluation. In the pre-processing phase, the raw data is explored and cleaned manually or through some unsupervised learning techniques. The processed data is expected to be valid, accurate, complete, consistent and uniform. After that, we can split the pre-processed data into a training dataset and test data which usually follow a ratio of $80 / 20$ (which is not necessary). In the model building phase, candidate models are built and trained by the training dataset. Different training techniques should be applied to achieve the least error rate. If the error is not converged, the models are usually modified by adding more neurons to make it wider, or adding more layers to make it deeper. If the training error converged, the trained network would be evaluated by the test 
dataset. The accuracy of the test dataset is the key metric to show whether we can deploy the program in a broader way. If the test accuracy is not high enough to deploy, it's not bad to repeat the model building and evaluating process with different groups of candidate models.

\section{Insights}

\subsection{The method for calculating accuracy.}

The test accuracy is a golden standard to evaluate the performance or training matureness of the DL program. However, the method to evaluate accuracy is doubted in some DL material research. The test accuracy is calculated by test data, which is never seen by the system during the training process. However, some researchers using training data and test data together to measure the accuracy of their system will overestimate the performance and lead to inaccurate results. ${ }^{[6,65,69]}$

\subsection{The dataset difference between material and IT}

Material science generates various data such as spectrum, abstracted or measured data points from instrument recorded curves $^{[85]}$, time series, images. ${ }^{[11]}$ The most sever problem is the total dimension of the material's property is large and always not intact. Some datasets describe electronic properties while others describe thermal properties. Even with a combination of multiple datasets, the problem of mismatch of data generation methods impacts the continuity and consistency. For example, the data from the experiment may not match the data from DFT, ab initio or atomistic simulation. The lack of continuity of this reduces the accuracy of the DL result Terminology is another gap between material scientists and computer scientists. For example, the term parameter could be the factor of varies in an experiment, however, it is actually the input data for deep learning scientists. On the contrary, DL scientist's parameter refers more to the so-called black box function parameter for the program.

\subsection{Advantage of GPU computing}

In the view of DL scientists, GPU is the essential tool for deep learning and the well-established framework (toolbox) also helps the researchers can confidently use GPU as their primary computation platform without extra training. DLs are computation-intensive tasks that can greatly speed up by parallel computing systems. A modern CPU consists of 4 or 8 parallel CPU cores, while a GPU generally consists of hundreds of simpler parallel cores. The parallel computing capability of GPU makes it super fast on matrix related operations, such as matrix multiplication. And those matrix related operations are also the dominant operations of deep learning tasks. A GPU can easily provide an order of magnitude or more speedup than a CPU of the same level for deep learning tasks. For example, the NVIDIA V100 GPU shows 32 times faster than state-of-the-art CPU on processing deep learning benchmarks. ${ }^{[86]}$ It may not be a problem to use a CPU for training a neural network if it has thousands of or millions of parameters. The tasks can be completed in hours or days using the CPU platform. However, the recently published GPT-3 model ${ }^{[87]}$ has 175 billion parameters, the training time needed by a single CPU could be hundreds of years.

\subsection{Toolbox}

The toolbox is widely used in DL studies due to its convenience. The major parts of the DL program remain the same and only a few layers change for specific projects or targets. In this case, it's better for material scientists to use a well-established DL toolbox rather than develop a new algorithm. It's DL scientists' job to improve the algorithm. Toolboxes such as PyTorch ${ }^{[88]}$, Keras ${ }^{[89]}$ provide user-friendly API and well-organized tutorial, which let the user without much knowledge can take advantage of the state-of-the-art deep learning techniques. ${ }^{[90-94]}$

\section{Deep learning and medical materials}

Among all applications of DL, the medical material might not be anything special or significant due to its small data quantity. More likely, the medical material becomes an extension of biomaterials ${ }^{[95,96]}$, biocompatible studies ${ }^{[97]}$, or biomedical oriented study sourced from general datasets. Such a situation is not likely to weaken the study of medical material, rather push the medical material field to be fused with other subjects and form some interdisciplinary ideas.

Recently, Wei Long Ng et al. ${ }^{[98]}$ reviewed the DL topic on $3 \mathrm{D}$ printed scaffolds, tissues and organs. As one of the hottest areas in materials science and engineering, the application of DL is still focusing on image recognition related subjects and which is also the most well-developed part in the DL field. ${ }^{[99,100]}$

Material properties are always in high dimensions, such as electrical properties, optical properties, mechanical properties, and thermal properties. Processing the high dimension and low sample size data is also challenging by using the DL algorithm. But DL algorithm designers are working in this field and designed a series of techniques. They defined those techniques as few-shot learning. ${ }^{[87,101]}$ As an emerging field, material scientists are suggested to follow up on new concepts and findings for the few-shot learning.

Data analysis is the easiest way for DL to participate in medical material. Similar to the image recognition technology which main hospitals and biotechnology companies are intensively studying ${ }^{[102,103]}$, acceleration of modeling, the failure/fatigue of medical material, aging of implant, crosssection design are chances for the DL tools. For example, conventional network analysis for conductive network cost high CPU time. ${ }^{[104]}$ DL tools could be designed for faster network analysis and reconstruction which enable high through-out computation quantity. ${ }^{[105]}$ As a result, the quality and reliability of the re-constructed network increases and which provides high quality percolation prediction.

Another possible field for DL is the combination with current computation methods such as $\mathrm{FEA}^{[79]}$, molecular 
modeling ${ }^{[73]}$ and DFT based modeling. ${ }^{[7]}$ Some studies used datasets generated from the aforementioned modeling methods, however, these datasets are too special to be extended for other studies. The key problem here is the generated data could not be incorporated into a general dataset. As a result, people may want to see an $a b$ initio based ceaseless supercomputer computing general properties of all chemicals or materials, such as electrical structure, vibrational property, thermal property. Such a project is cost ineffective cause most of the data could be useless if they are not directly being used. Frankly, it's more like simulating all molecules in the air to predict the weather 10 years later. So, how to balance the need for the generation of data and future research is one point to be considered.

Besides, screening of all possible materials could be another main use of DL in medical material. For example, a polymer's properties change with their repeating units, chain length, branch structure, additives, thermal history and many other conditions. The laboratory accessible raw polymer may not adapt to a new study. In the old fashion, we would try some more similar polymers or change its composition based on our need and we all know this is not effective. Mostly, we just make compromises with the available material. With the application of a DL toolbox or based on well-established databases, an optimized solution could be produced.

\section{Summary and perspective}

DL method has been widely used for many fields while materials science and engineering are quite slow on catching up due to the imperfect nature of the material dataset. As we have discussed the different thinking routes between DL scientists and material scientists. Also, we have introduced different networks. As a summary, we organized the networks from deep learning science with their application in material science in Fig. 4.

In order to obtain an accurate, fast and reliable result, there are still many problems to conquer, such as a systematic database or standard of data form and storing method. Looking back to the evolution of DL, one of the keys is sufficient data to be available for training, both for the program and the researchers. The premise of using machine learning, DL related methods is a well labeled dataset that requires assistance from data science. Google's Research Director, Peter Norving, claiming that "We don't have better algorithms. We just have more data. More data beats clever algorithm, but better data beats more data." ${ }_{[106]}$

At last, a perspective on how material scientists should participate in the racing of artificial intelligence is provided for reference. Two key aspects should be pay attention in future materials research:

i) Applying DL toolbox into materials science and engineering in an appropriate way;

ii) Establish well defined datasets with cleaned entries.

Current materials-DL studies show a lack of maturity, it seems the way to apply DL tool into materials science and engineering has not been paved well. With the idea of DL being broadcasted, more researchers would attempt their subject with DL tools. ${ }^{[107]}$ In this case, we should keep good communication with DL scientists for the newest ideas, findings and especially DL toolboxes. Interdisciplinary collaboration is always difficult especially for those with a huge knowledge barrier. The introduction of DL concepts for

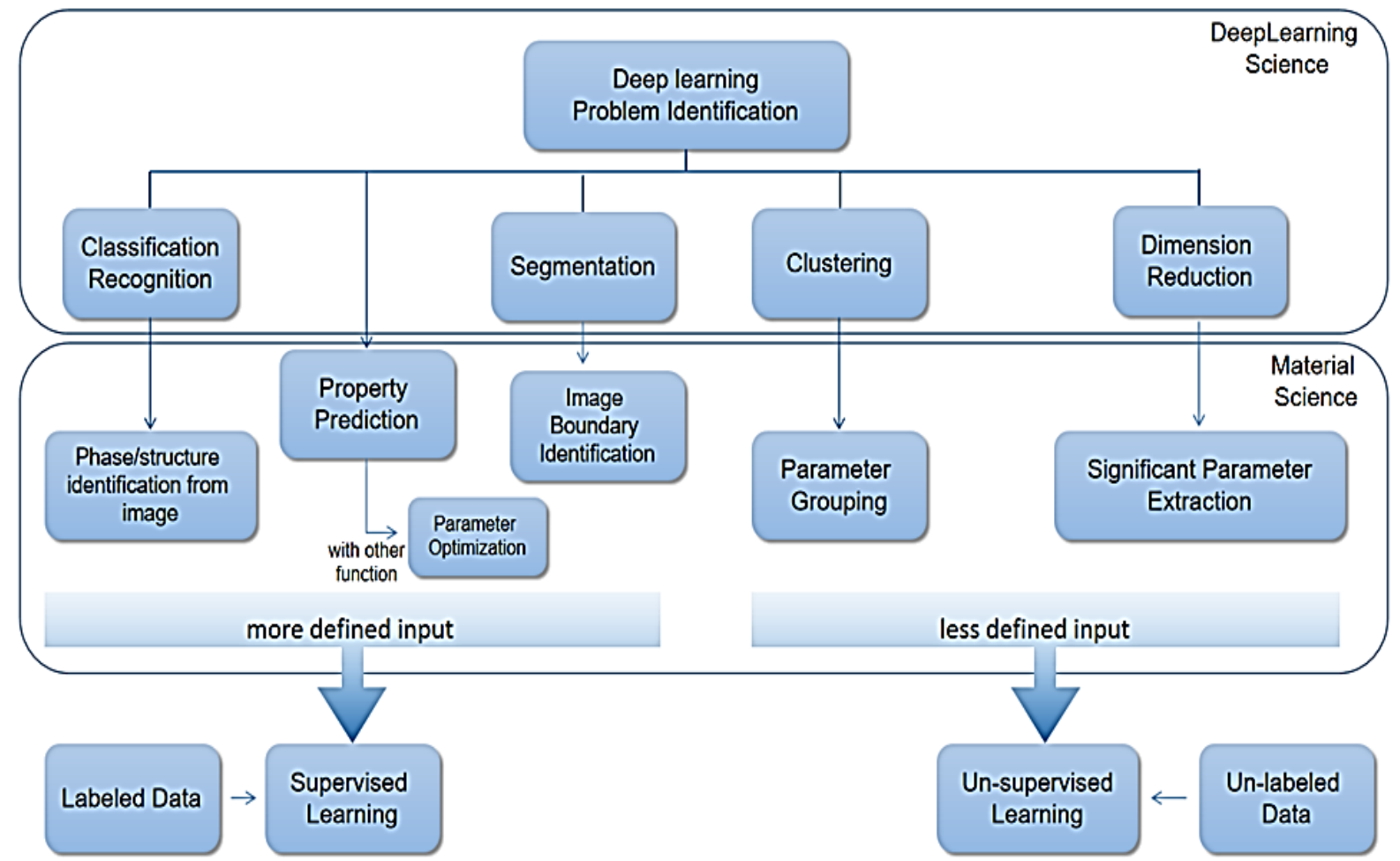

Fig. 4 Deep learning problem identification and application in material science and engineering master chart. 
undergraduate students may be a smart start. Second, in the coming years, we are expecting a burst in data size materials science and engineering. In this case, material scientists have the responsibility to organize these data systematically and make them ready for DL toolboxes. In short, the establishment of well-organized material science and engineering dataset will promote future DL applications at an unexpected speed. Although the quality of these research may not be helpful for our conservative material science, they are pushing the need and step on establishing a generalized dataset for DL. Overall, we should carefully consult our DL specialists while introducing it into our research and be ambitious on using new technology for accelerating our research. ${ }^{[108]}$

\section{Conflict of Interest}

There is no conflict of interest.

\section{Supporting Information}

Not applicable

\section{References}

[1] G. A. Van Norman, JACC: Basic Transl. Sci. 2016, 3, 170179, doi: 10.1016/j.jacbts.2016.03.002.

[2] G. A. Van Norman, JACC: Basic Transl. Sci. 2016, 3, $277-$ 287, doi: 10.1016/j.jacbts.2016.03.009.

[3] D. M.Beazley, P. S. Lomdahl, N. Grønbech-Jensen, R. Giles, and P. Tamayo, Аnnu. Rev. Comput. Phys. III, 2017, 119-175, doi: 10.1142/9789812830647_0004.

[4] F. Ricci, W. Chen, U. Aydemir, G. J. Snyder, G. Rignanese, A. Jain, and G. Hautier, Sci. Data, 2017, 4, 170085, doi: 10.1038/sdata.2017.85.

[5] C. P. Callaway, K. Hendrickson, N. Bond, S. M. Lee, P. Sood, and S. S. Jang, ChemPhysChem, 2018, 19, 1655-1664, doi: 10.1002/cphc.201701337.

[6] C. Chen, and G. X. Gu, MRS Commun. 2019, 9, 556-566, doi: 10.1557/mrc.2019.32.

[7] J. Li, T. Chen, K. Lim, L. Chen, S. A. Khan, J. Xie, and X. Wang, Adv. Intell. Syst. 2019, 3, 1900029, doi: 10.1002/aisy.201900029.

[8] S. Targ, D. Almeida, and K. Lyman, ArXiv preprint, 2016 arXiv: 1603.08029 .

[9] D. Silver, J. Schrittwieser, K. Simonyan, I. Antonoglou, A. Huang, A. Guez, T. Hubert, L, Baker, M. Lai, A. Bolton, Y. Chen, T. Lillicrap, F. Hui, L. Sifre, G. van den Driessche, T. Graepel and D. Hassabis, Nature, 2017, 550, 354-359, doi: 10.1038/nature24270.

[10] Y. LeCun, Y. Bengio, and G. Hinton, Nature, 2015, 521, 436444, doi: 10.1038/nature14539.

[11] Q. Rong, H. Wei, and H. Bao. SSRN, 2019, 3358857.

[12] M. Cho, J. H. Lee, S. Kim, J. S. Kim, and S. Timilsina, ACS Appl. Mater. Interfaces, 2019, 11, 11910-11919, doi: 10.1021/acsami.9b00464.

[13] Z. Yang, X. Li, L. C. Brinson, A. N. Choudhary, W. Chen, and A. Agrawal, J. Mech. Des., 2018, 140, 111416, doi: 10.1115/1.4041371.
[14] X. Li, Z. Yang, L. C. Brinson, A. N. Choudhary, A. Agrawal, and W. Chen, in International Design Engineering Technical Conferences and Computers and Information in Engineering Conference, ASME, 2018, 2B, 85633, V02BT03A008, doi: 10.1115/DETC2018-85633.

[15] J. Brownlee. A gentle introduction to mini-batch gradient descent and how to configure batch size. ML Mastery. Viewed on Feb. 072021.

[16] I. Sutskever, J. Martens, G. Dahl, and G. Hinton, in International Conference on Machine Learning, PMLR, 2013, 28, 1139.

[17] J. Duchi, E. Hazan, and Y. Singer, J. Mach. Learn. Res., 2011, 12, 2121, http://jmlr.org/papers/v12/duchi11a.html.

[18] M. D. Zeiler, ArXiv preprint, 2012, arXiv:1212.5701.

[19] T. Tieleman, and G. Hinton, COURSERA: Neural networks for machine learning 2012, 4, 26-31. https://www.cs.toronto.edu/ hinton/coursera/lecture6/lec6.pdf [20] D. P. Kingma, and J. Ba. ArXiv preprint, 2014, arXiv: 1412.6980.

[21] N. Srivastava, G. Hinton, A. Krizhevsky, I. Sutskever, and R. Salakhutdinov. J. Mach. Learn. Res., 2014, 15, 1929-1958.

[22] S. Ioffe, and C. Szegedy, ArXiv preprint, 2015, arXiv: 1502.03167.

[23] S. Ruder, ArXiv preprint, 2016, arXiv:1609.04747.

[24] A. Krizhevsky, I. Sutskever, and G. Hinton, Commun. ACM, 2017, 60, 84-90, doi: 10.1145/3065386.

[25] C. Szegedy, W. Liu, Y. Jia, P. Sermanet, S. Reed, D. Anguelov, D. Erhan, V. Vanhoucke, and A. Rabinovich, in Proceedings of the IEEE conference on Computer Vision and Pattern Recognition (CVPR), 2015, 1-9, doi: 10.1109/CVPR.2015.7298594.

[26] K. He, X. Zhang, S. Ren, and J. Sun, In Proceedings of the IEEE conference on Computer Vision and Pattern Recognition (CVPR), 2016, 770, doi: 10.1109/CVPR.2016.90.

[27] O. Russakovsky, J. Deng, H. Su, J. Krause, S. Satheesh, S. Ma, Z. Huang, A. Karpathy, A. Khosla, M. Bernstein and A. C. Berg, Int. J. Comput. Vis., 2015, 115, 211-252, doi: 10.1007/s11263-015-0816-y.

[28] Y. Li, H. Yan, and Y. Zhang, in 2019 IEEE 17th International Conference on Industrial Informatics (INDIN), 2019, 1, 17351740, doi: 10.1109/INDIN41052.2019.8972334.

[29] S. Hochreiter, and J. Schmidhuber, Neural Comput., 1997, 9, 1735-1780, doi: 10.1162/neco.1997.9.8.1735.

[30] K. Cho, B. Van Merriënboer, C. Gulcehre, D. Bahdanau, F. Bougares, H. Schwenk, and Y. Bengio, ArXiv preprint, 2014, arXiv: 1406.1078 .

[31] J. Zhang, P. Wang, and R. X. Gao, Compute. Ind., 2019, 107, 11-21, doi: 10.1016/j.compind.2019.01.011.

[32] I. Goodfellow, J. Pouget-Abadie, M. Mirza, B. Xu, D. Warde-Farley, S. Ozair, A. Courville, and Y. Bengio, in Proceedings of the 27th International Conference On Neural Information Processing Systems, 2014, 2672-2680, doi: $10.5555 / 2969033.2969125$.

[33] H. Dong, W. Hsiao, L. Yang, and Y. Yang, ArXiv preprint, 2017, arXiv: 1709.06298. 
[34] H. Wu, S. Zheng, J. Zhang, and K. Huang. in Proceedings of the 27th ACM International Conference on Multimedia, 2019, 2487-2495, doi: 10.1145/3343031.3350944.

[35] A. Ghosh, B. Bhattacharya, and S. B. R. Chowdhury, arXiv preprint, 2016, arXiv: 1611.08788.

[36] A. Radford, L. Metz, and S. Chintala, ArXiv preprint, 2015. arXiv:1511.06434.

[37] ICSD - the world's largest database for completely identified inorganic crystal structures. Viewed on 07 Feb 2021, https://icsd.products.fiz-karlsruhe.de/.

[38] The open quantum materials database. Viewed on $07 \mathrm{Feb}$ 2021, http://oqmd.org/.

[39] S. Bell, P. Upchurch, N. Snavely, K. Bala, Computer Vision and Pattern Recognition, 2015. Viewed on 07 Feb 2021, http://opensurfaces.cs.cornell.edu/publications/minc/.

[40] Automatic-FLOW for Materials Discovery. Viewed on 07 Feb 2021, http://www.aflowlib.org/search/apool.php.

[41] Novel Materials Discover Centre of Excellence. Viewed on 07 Feb 2021, https://nomad-coe.eu/.

[42] Quantum-Machine datasets. Viewed on $07 \mathrm{Feb} 2021$, http://quantum-machine.org/datasets/.

[43] The EPiC database. Viewed on 07 Feb 2021, https://msd.unimelb.edu.au/research/projects/current/environme ntal-performance-in-construction/epic-database.

[44] NanoMine: an Online Platform of Materials Genome Prediction for Polymer Nanocomposites. Viewed on 07 Feb 2021, http://brinsonlab.pratt.duke.edu/research/mgp/Nanomine.

[45] Polymer Property Predictor and Database, CHiMaD. Viewed 07 Feb 2021, https://pppdb.uchicago.edu/.

[46] The Materials Project. Viewed on 07 Feb 2021, https://www.materialsproject.org/.

[47] Khazana ${ }^{1.0}$ : A Computational Materials Knowledgebase. Viewed on 07 Feb 2021.

[48] Active thermochemical tables. Viewed on 07 Feb 2021, https://atct.anl.gov/.

[49] Inorganic Material Database (AtomWork). Viewed on 07 Feb 2021, https://crystdb.nims.go.jp/en/.

[50] Thermochemical Tables. Viewed on 21 Dec 2020, https://janaf.nist.gov/.

[51] Index of Material Properties. Viewed on 07 Feb 2021, https://trc.nist.gov/cryogenics/materials/materialproperties.htm

[52] NIST Property Data Summaries for Advanced Materials. Viewed on 07 Feb 2021.

[53] S. Chmiela, H. E. Sauceda, K. Müller, and A. Tkatchenko, Nat. commun., 2018, 9, 1-10, doi: 10.1038/s41467-018-06169-2.

[54] C. Sutton, L. M. Ghiringhelli, T. Yamamoto, Y. Lysogorskiy, L. Blumenthal, T. Hammerschmidt, J. R. Golebiowski, X. Liu, A. Ziletti, and M. Scheffler, npj Comput. Mater., 2019, 5, 111, doi: https://doi.org/10.1038/s41524-019-0239-3.

[55] C. Nyshadham, M. Rupp, B. Bekker, A. V. Shapeev, T. Mueller, C. W. Rosenbrock, G. Csányi, D. W. Wingate, and G. LW Hart, npj Comput. Mater., 2019, 5, 51, doi: 10.1038/s41524019-0189-9

[56] W. J. Szlachta, A. P. Bartók, and G. Csányi, Phys. Rev. B, 2014, 90, 104108, doi: 10.1103/PhysRevB.90.104108.
[57] L. J. Nelson, V. Ozoliņš, C. S. Reese, F. Zhou, and G. LW Hart, Phys. Rev. B, 2013, 88, 155105, doi: 10.1103/PhysRevB.88.155105.

[58] A. P. Bartók, M. J. Gillan, F. R. Manby, and G. Csányi, Phys. Rev. B, 2013, 88, 054104, doi: 10.1103/PhysRevB.88.054104.

[59] F. Ricci, W. Chen, U. Aydemir, G. J. Snyder, G. Rignanese, A. Jain, and G. Hautier, Sci. Data, 2017, 4, 170085, doi: 10.1038/sdata.2017.85.

[60] A catalog of publicly available NASA Datasets. Viewed on $07 \mathrm{Feb} 2021$, https://nasa.github.io/data-nasa-gov-frontpage/.

[61] NIST public data catalog. Viewed on 07 Feb 2021, https://www.nist.gov/data.

[62] The databases by Office of Environment, Health, Safety \& Security of the Department of Energy. Viewed on 07 Feb 2021, https://www.energy.gov/ehss/corporate-reporting-

analysis/databases.

[63] Office of Scientific and Technical Information of the U.S. Department of Energy. Viewed on 07 Feb 2021, https://www.osti.gov/.

[64] The Materials Data Facility (MDF). Viewed on $07 \mathrm{Feb} 2021$, https://materialsdatafacility.org/.

[65] A. Jain, S. P. Ong, G. Hautier, W. Chen, W. D. Richards, S. Dacek, S. Cholia, D. Gunter, D. Skinner, G. Ceder, and K. A. Persson. APL Mater., 2013, 1, 011002, doi: 10.1063/1.4812323.

[66] National Institute for Materials Science Databases. Viewed on $07 \mathrm{Feb} 2021$.

[67] National Science \& Technology Infrastructure. Viewed on 07 Feb 2021, http://nsdata.cn.

[68] A. Soualy, A. Walter, O. Sutter, and J. Nault, Clin. Res. Hepatol. Gastroenterol., 2020, 44, e25-e28, 10.1016/j.clinre.2019.06.016.

[69] R. Loffroy, T. Mouillot, M. Bardou, and O. Chevallier, Expert Rev. Gastroenterol. Hepatol., 2020, 14, 975-984, doi: 10.1080/17474124.2020.1790355.

[70] J. Li, and X. Wang, APL Mater., 2017, 5, 073801, doi: 10.1063/1.4978936.

[71] Z. Jin, Z. Zhang, and G. X. Gu, Manuf. Lett., 2019, 22, 1115, doi: 10.1016/j.mfglet.2019.09.005.

[72] P. Wang, R. X. Gao, and R. Yan, CIRP Ann., 2017, 66, 429432, doi: 10.1016/j.cirp.2017.04.013.

[73] S. Ye, B. Li, Q. Li, H. Zhao, and X. Feng, Appl. Phys. Lett., 2019, 115, 161901., doi: 10.1063/1.5124529.

[74] J. Zhang, P. Wang, and R. X. Gao, Comput. Ind., 2019, 107, 11-21, doi: 10.1016/j.compind.2019.01.011.

[75] Z. Zhang, I. Fidan, and M. Allen, Inventions, 2020, 5, 25, doi: 10.3390/inventions5030025.

[76] X. Liu, F. Gasco, J. Goodsell, and W. Yu, Compos. Struct., 2019, 230, 111505, doi: 10.1016/j.compstruct.2019.111505.

[77] Z. Yang, Y. C. Yabansu, D. Jha, W. Liao, A. N. Choudhary, S. R. Kalidindi, and A. Agrawal, Acta Mater., 2019, 166, 335345, doi: 10.1016/j.actamat.2018.12.045.

[78] M. Mozaffar, R. Bostanabad, W. Chen, K. Ehmann, J. Cao, and M. A. Bessa, Proc. Natl. Acad. Sci., 2019, 116, 26414-26420, doi: 10.1073/pnas.1911815116. 
[79] R. Anderson, A. Biong, and D. A. Gómez-Gualdrón, J. Chem. Theory Comput., 2020, 16, 1271-1283, doi: 10.1021/acs.jctc.9b00940.

[80] S. So, J. Mun, and J. Rho, ACS Appl Mater. Interfaces, 2019, 11, 24264-24268, doi: 10.1021/acsami.9b05857.

[81] J. Li, T. Chen, K. Lim, L. Chen, S. A. Khan, J. Xie, and X. Wang, Adv. Intell. Syst., 2019, 1, 1900029, doi: 10.1002/aisy.201900029.

[82] A. Nasiri, J. Bao, D. Mccleeary, S. M. Louis, X. Huang and J. Hu, IEEE Access, 2019, 140534-140541, doi: 10.1109/ACCESS.2019.2943210.

[83] R. Lorenzoni, I. Curosu, S. Paciornik, V. Mechtcherine, M. Oppermann, and F. Silva, Cem. Concr. Compos., 2020, 108, 103551, doi: 10.1016/j.cemconcomp.2020.103551.

[84] A. Khan, D. Ko, S. C. Lim, and H. S. Kim, Compos. B. Eng., 2019, 161, 586-594, 10.1016/j.compositesb.2018.12.118.

[85] A. A. Guda, S. A. Guda, A. Martini, A. L. Bugaev, M. A. Soldatov, A. V. Soldatov, and C. Lamberti, Radiat. Phys. Chem., 2020, 175, 108430, doi: 10.1016/j.radphyschem.2019.108430.

[86] NVIDIA V100 Tensor Core GPU. Viewed on 07 Feb 2021, https://www.nvidia.com/en-us/data-center/v100/.

[87] T. B. Brown, B. Mann, N. Ryder, M. Subbiah, J. Kaplan, P. Dhariwal, A. Neelakantan, et al, ArXiv preprint, 2020, arXiv:2005.14165.

[88] A. Paszke, S. Gross, F. Massa, A. Lerer, J. Bradbury, G. Chanan, T. Killeenl, ArXiv preprint, 2019, arXiv:1912.01703.

[89] F. Chollet, and others, 2015. Viewed on 07 Feb 2021, https://keras.io.

[90] D. Jha, L. Ward, A. Paul, W. Liao, A. Choudhary, C. Wolverton, and A. Agrawal, Sci. Rep., 2018, 8, 1-13, doi: 10.1038/s41598-018-35934-y.

[91] L. Lu, M. Dao, P. Kumar, U. Ramamurty, G. E. Karniadakis, and S. Suresh, Proc. Natl. Acad. Sci., 2020, 117, 7052-7062, doi: 10.1073/pnas.1922210117.

[92] K. T. Schutt, P. Kessel, M. Gastegger, K. A. Nicoli, A. Tkatchenko, and K-R. Müller, J. Chem. Theory Comput., 2018, 15, 448-455, doi: 10.1021/acs.jctc.8b00908.

[93] Z. Liu, C. T. Wu, and M. Koishi, Comput. Methods Appl. Mech. Eng., 2019, 345, 1138-1168, doi: 10.1016/j.cma.2018.09.020.

[94] J. Lee, W. B. Park, J. H. Lee, S. P. Singh, and K. Sohn, Nat. commun., 2020, 11, 86, doi: 10.1038/s41467-019-13749-3.

[95] A.B.M. S. Hossain, M. M. Uddin, V. N. Veettil, and M. Fawzi, Data Brief, 2018, 17, 162-168, doi: 10.1016/j.dib.2017.12.046.

[96] P. N. Schofield, U. Kulka, S. Tapio, and B. Grosche, Int. J. Radiat. Biol., 2019, 95, 861-878, doi: 10.1080/09553002.2019.1589026

[97] T. L. Tepla, I. V. Izonin, Z. A. Duriagina, R. O. Tkachenko, A. M. Trostianchyn, I. A. Lemishka, V. V. Kulyk and T. M. Kovbasyuk, Archives Mater. Sci. Eng., 2018, 93, 32-40, doi: 10.5604/01.3001.0012.6944.

[98] W. L. Ng, A. Chan, Y. S. Ong, and C. K. Chua, Virtual Phys. Prototyp., 2020, 3, 340-358, doi: $10.1080 / 17452759.2020 .1771741$.
[99]S. Masubuchi, E. Watanabe, Y. Seo, S. Okazaki, T. Sasagawa, K. Watanabe, T. Taniguchi, and T. Machida, npj 2D Mater. Appl., 2020, 4, 3, doi: 10.1038/s41699-020-0137-z.

[100] E. Zhang, Mi. Yin, and G. E. Karniadakis, ArXiv preprint, 2020, arXiv:2009.04525.

[101] Y. Wang, Q. Yao, J. Kwok and L. M. Ni, ACM Comput. Surv. 2020, 53, 63, doi: 10.1145/3386252.

[102] B. Lou, S. Doken, T. Zhuang, D. Wingerter, M. Gidwani, N. Mistry, L. Ladic, A. Kamen, and M. E. Abazeed, Lancet Digit. Health, 2019, 1, e136-e147, doi: 10.1016/S2589-7500(19)300585.

[103] L. Lu, Y. Zheng, G. Carneiro, and L. Yang, Deep learning and convolutional neural networks for medical image computing. Switzerland: Springer International Publishing, 2017, doi: 10.1007/978-3-319-42999-1.

[104] N. Al Habis, Nuha, C. Liu, J. Dumuids, and K. Lafdi, RSC Adv., 2016, 6, 95010-95020, doi: 10.1039/C6RA19477J.

[105] X. Zeng, S. Zhu, X. Liu, Y. Zhou, R, Nussinov, and F. Cheng, Bioinform., 2019, 35, 5191-5198, doi: 10.1093/bioinformatics/btz418.

[106] A. Halevy, P. Norvig, and F. Pereira. IEEE Intel. Syst., 2009, 24, 8-12, doi: 10.1109/MIS.2009.36.

[107] C. Yang, Y. Kim, S. Ryu, and G. X. Gu, MRS Commun., 2019, 9, 609-617, doi: 10.1557/mrc.2019.49.

[108] O. Hakimi, M. Krallinger, and M. Ginebra, Nat. Rev. Mater., 2020, 5, 553-556, doi: 10.1038/s41578-020-0215-z.

\section{Author information}

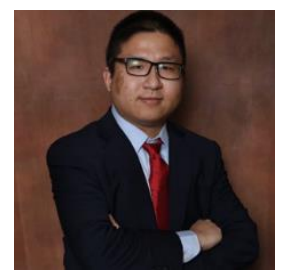

Yangjie Qi received the B.S. (2012) from the Dept. of Electrical Engineering, Anhui Univ., Hefei, China, and the M.S. (2015) from the Dept. of Electrical and Computer Engineering from Univ. of Dayton, $\mathrm{OH}$. He is currently pursuing his Ph.D. in the Univ. of Dayton. His Ph.D. research work focuses on the design of low power, high performance multicore architectures for deep learning.

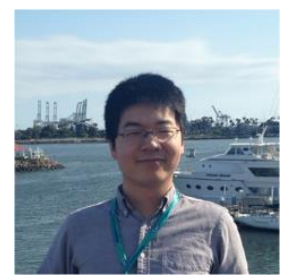

Chang Liu received his B.S. (2012) in Polymer from the Beijing University of Chemical Technology, Beijing, China. Then he joined the Institute of Chemistry, Chinese Academy of Science (ICCAS) in 2012. He obtained his M.S. (2014) and Ph.D. (2019) in the University of Dayton, OH. Later he joined the Lerner Research Institute of the Cleveland Clinic in 2020. He established the Adva-Nano LLC as a co-founder in 2020. His research includes polymers, characterization, additive manufacturing, biomedical materials, carbon nanomaterials, structural health monitoring topics.

Publisher's Note Engineered Science Publisher remains neutral with regard to jurisdictional claims in published maps and institutional affiliations. 